\title{
Acute Respiratory Distress Syndrome
}

\author{
Hsing l. Chen ${ }^{a}$ Shang Jyh Kao ${ }^{b}$ David Wang $^{c}$ Ru Ping Lee ${ }^{d}$ Chain Fa Su ${ }^{e}$ \\ alnstitute of Medical Sciences, Tzu Chi University, Hualien, bDepartment of Internal Medicine, \\ Shin Kong Wu Ho-Su Memorial Hospital, Taipei, ${ }^{c}$ Department of Nursing, Tzu Chi College of Technology, \\ Institute of Medical Sciences and Department of Nursing, ${ }^{e}$ Department of Neurosurgery, \\ Tzu Chi Hospital and University, Hualien, Taiwan, ROC
}

\section{Key Words}

Pulmonary edema . Acute respiratory distress

syndrome $\cdot$ Lung injury $\cdot$ Nitric oxide

\begin{abstract}
Acute respiratory distress syndrome (ARDS) can be associated with various disorders. Among these, coronavirus infection may cause life-threatening severe acute respiratory syndrome (SARS). In this review, we present animal models and techniques for the study of ARDS, and discuss the roles and possible mechanisms of various chemical factors, including nitric oxide (NO). Our early work revealed that cerebral compression elicits severe hemorrhagic pulmonary edema (PE), leading to central sympathetic activation that results in systemic vasoconstriction. The consequence of systemic vasoconstriction is volume and pressure loading in the pulmonary circulation. Vasodilators, but not oxidant radical scavengers, are effective in the prevention of centrogenic PE. In isolated perfused lung, exogenous and endogenous NO enhances lung injury following air embolism and ischemia/reperfusion. In contrast, NO synthase (NOS) inhibitors reverse such lung injury. Although NO is important in maintaining vasodilator tone, hypoxiainduced pulmonary vasoconstriction is accompanied by
\end{abstract}

an increase instead of a decrease in NO release. In animal and isolated lung studies, endotoxin produces acute lung injury that is associated with increases in cytokines and inducible NOS mRNA expression, suggesting that NO is toxic to the lung in endotoxin shock. Recently, we reported several rare cases that indicate that ARDS in patients with Japanese $B$ encephalitis, lymphangitis with breast cancer and fat embolism is caused by different mechanisms. Our early and recent studies on ARDS and $P E$ may provide information for clinical practice and the understanding of the pathogenesis of SARS.

Copyright $\odot 2003$ National Science Council, ROC and S. Karger AG, Basel

\section{Introduction}

The recent outbreak of the life-threatening severe acute respiratory syndrome (SARS) in many countries has resulted in deaths of patients infected by coronavirus $[22$, $23,28,34]$. In addition to SARS, acute respiratory distress syndrome (ARDS) can be induced by various disorders. Accordingly, investigations of the mechanism of ARDS can further our understanding of the yet unknown mechanism of SARS. In this brief review, we present early and recent studies in animal and human subjects with either ARDS or acute pulmonary edema (PE).

\section{KARGER}

Fax +41613061234

E-Mail karger@karger.eh

www.karger.com

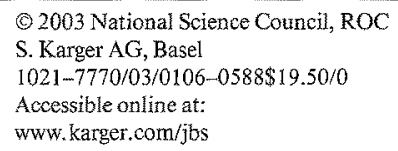


A series of studies on neurogenic PE resulting from cerebral compression or intracranial hypertension suggest that brain injuries initiate massive central sympathetic activation. This, in turn, causes systemic vasoconstriction and shift of blood from the systemic circulation to the lung $[2-4,6,8,9,11,12,14]$. In an isolated lung that is subject to air embolism, ischemia/reperfusion (I/R) or endotoxin, nitric oxide (NO) is detrimental to lung tissues and causes acute injury, although it reduces pulmonary hypertension [21, 24, 36]. Recently, we reported [19] that in patients with diseases of rare causes, ARDS is induced by several other mechanisms. In patients with Japanese B encephalitis, the viral infection destroys depressor areas in the medulla, implying that central sympathetic activation might be the cause of ARDS. On the other hand, NO, cGMP and serotonin are possibly involved in ARDS due to fat embolism. We suggest that further investigation of the involvement of $\mathrm{NO}$, central nervous lesions and other chemical factors will advance our understanding of SARS caused by coronavirus infection.

\section{Animal Models and Techniques for ARDS}

Our early studies used whole animals (mainly rats of the Sprague-Dawley strain) to investigate the mechanism of neurogenic PE induced by cerebral compression [2-14]. Right and left heart bypass were employed to elucidate the importance of cardiac output and venous return in the genesis of pulmonary pathology [8]. A technique of regional weighing described by Fell and Rushmer [15] was used to demonstrate the shift of blood volume from the systemic circulation to the lung $[2,9-11,14]$. The total heart bypass technique has allowed us to perfuse the systemic and pulmonary circulation with constant flow. We examined systemic and pulmonary hemodynamics in response to intracranial hypertension and found that intracranial hypertension causes an increase in systemic and pulmonary vascular resistance and a decrease in capacity [13]. Spectral analysis for the assessment of steady and pulsatile hemodynamics demonstrated that intracranial hypertension causes an increase in characteristic impedance, wave reflection and total peripheral resistance, along with a decrease in cardiac output [32]. In Sprague-Dawley rats, endotoxin causes systemic hypotension with an increase in cytokine and inducible NO synthase (iNOS) mRNA expression in the injured lung. These changes are attenuated by NOS inhibitors such as $\mathrm{N}^{\omega}{ }^{\omega}$-nitro- $L$-arginine methyl ester (L-NAME), $\mathrm{S}, \mathrm{S}^{\prime}-1,4-$ phenylene-bis-(1,2-ethanedinyl) bis-isothiourea dihydrobromide and dexamethasone [37].
A good preparation for the study of acute lung injury is isolated lungs in situ $[17,18,21]$. A previous method involved removing the isolated lung from the body and placing it on a force-displacement transducer to record the change in lung weight. Because this procedure is rather complicated and unstable, we invented a device for measuring the change in lung weight by a scale. The isolated perfused lung is not removed from the body. Instead, the whole body is placed on a scale platform and a digital-analogue converter records the change in lung weight on a recorder. In the isolated lung preparation, lung weight gain, lung weight/body weight ratio, microvascular permeability and protein concentration in bronchoalveolar lavage could be measured and calculated to evaluate the extent of lung injury, as well as to determine the pulmonary vascular tone.

Wang et al. [36] found that in an isolated lung, $L$-arginine and inhaled NO increased lung injury induced by air embolism, while L-NAME attenuated it. Mecaprine, an agent that reduces oxygen free radicals, also protects the lung from acute injury following I/R [29]. It was surprising to find that hypoxia-induced vasoconstriction is associated with an increased release of NO from the lung [35]. This observation is in contrast with the notion that NO is a vasodilator and that the reduction of NO may lead to vasoconstriction. In endotoxic shock, NO was shown to be harmful to the lung, and the major site of NO formation is in the lung, not the organs [24]. NO also mediates lung damage following $\mathrm{I} / \mathrm{R}$. $L$-Arginine and inhaled NO enhance $\mathrm{I} / \mathrm{R}$-induced $\mathrm{PE}$, while NOS inhibitors reverse I/R lung injury [21].

A recently developed animal model of conscious and unrestrained rats is now available [26]. With this model, the condition of the animals can be observed and arterial pressure, heart rate and biochemical factors can be measured for more than $72 \mathrm{~h}$ without anesthesia. We used this novel model to investigate the physiological and chemical indicators for early and late stages of sepsis-associated pulmonary injury [25]. Extensive studies using this technique have been carried out in our laboratory.

\section{Chemical Factors and Involvement of NO}

PE induced by cerebral compression is not affected by cholinergic blockers (atropine) or beta-adrenergic blockers (propranol). However, it is prevented by $\alpha$-adrenergic blocking agents (phentolamine and phenoxybenzamine), norepinephrine depleter (respine) and a sympathetic neuronal blocker (bretylium). High doses of intravenous nor- 
epinephrine, angiotensin and vasopressin induce severe systemic vasoconstriction and $P E[4]$.

Previous studies have demonstrated that vasodilators such as nitroprusside and nitroglycerin cause a dosedependent attenuation of neurogenic PE, while oxidant scavengers such as dimethylthiourea and superoxide dismutase exert no significant effect on centrogenic PE [7]. $\mathrm{NO}$ is an important substance in the regulation of arterial pressure $[5,6,20,27,30]$. Since NO is a free radical, however, it may lead to the formation of hydroxyl radical and peroxynitrite, which are toxic to many organs including the lung $[27,33,38]$. Thus, the effects of exogenous and endogenous $\mathrm{NO}$ were investigated in our recent studies on lung injury induced by various disorders.

In Sprague-Dawley rats, endotoxin produces systemic hypotension, accompanied by enhancement of cytokines (tumor necrosis factor and interleukins) and iNOS mRNA expression [37]. In isolated perfused lung, exogenous and endogenous NO is detrimental to the lung following air embolism and $\mathrm{I} / \mathrm{R}[29,36]$. The endotoxininduced ARDS was found to be caused by large amounts of NO release in the lung [24]. The I/R-induced lung injury is exacerbated by NO inhalation and $L$-arginine administration, but is attenuated by an NOS inhibitor, L-NAME [21]. In patients with fat embolism due to long bone fracture, the plasma levels of cGMP and nitrate/nitrite are significantly increased, suggesting that $\mathrm{NO}$ is involved in ARDS associated with fat embolization [19].

\section{Possible Mechanisms}

Our early work indicated that massive sympathetic drive is responsible for the pathogenesis of neurogenic PE. $P E$ induced by cerebral compression is not affected by vagotomy and adrenalectomy, but is prevented by spinal transaction $[12,14]$. In rats under monitoring of aortic and pulmonary blood flow, cerebral compression causes a dramatic decrease in aortic flow (cardiac output), to less than $50 \%$ of the basal value. The pulmonary flow then slowly declines. We showed that the imbalance in right and left cardiac output resulted in the accumulation of blood in the lung [8]. Furthermore, we used right and left heart bypass in that study. In the left heart bypass, aortic flow was kept constant. The lung change was not discernible following cerebral compression. In the right heart bypass, the pulmonary flow was constant and the left cardiac output was allowed to change. Cerebral compression produced severe $\mathrm{PE}$. These findings suggest that the decrease in left ventricular output is the major cause of
PE. Venous return, however, is not essential. In another study, regional sympathectomy was performed to elucidate the participation of various vascular beds in neurogenic PE [10]. Splanchnic sympathectomy by dissection of the splanchnic nerves and celiac plexus almost completely prevented PE induced by cerebral compression. On the other hand, pulmonary sympathetic denervation by excising the thoracic sympathetic chains from the stellate ganglia to $\mathrm{T} 4$ attenuated the extent of PE by approximately $30 \%$. These results suggest that sympathetic splanchnic vasoconstriction is essential for the production of neurogenic PE and that pulmonary sympathetic vasoconstriction is not the major factor involved. When the lung is distended by accumulation of blood volume, pulmonary vasoconstriction can, however, cause an additional effect to enhance the pulmonary venous hypertension and PE.

Hypoxia-induced pulmonary vasoconstriction is a well-known phenomenon. The physiological significance is that capillary flow to hypoxic alveoli can be reduced, and the flow is thus shifted to oxygenated alveoli. It has been speculated that inadequate NO formation may be responsible for the increased vascular tone, pulmonary vasoconstriction and hypertension [for reviews, see ref. 6 , 27]. This contention was challenged by a study from our laboratory [35]. An NO detector probe was placed in the pulmonary vein in isolated perfused lung. Real-time NO monitoring revealed that NO release was increased, accompanied by pulmonary arterial hypertension following hypoxia. $L$-Arginine was able to potentiate NO release and reduce the pulmonary hypertension, while L-NAME produced opposite effects. These findings suggest that continuous NO release from the lung maintains pulmonary arterial pressure and that insufficient $\mathrm{NO}$ formation is not the cause of hypoxia-induced pulmonary hypertension. In this connection, studies from our laboratory $[5,6$, $20]$ also contest the early consensus that impairment of endothelial function and NO formation is the cause of hypertension due to reduction of the vasodilatory effect of NO [for a review, see ref. 27].

Pulmonary arterial pressure, microvascular permeability, lung weight/body weight ratio and protein concentration in bronchoalveolar lavage have been measured or calculated before and after air embolism, I/R and endotoxin administration in isolated perfused lung. NO only exerts a beneficial effect on the increase in pulmonary arterial pressure (pulmonary hypertension). This substance mediates the induction of acute lung injury induced by air embolism, I/R and endotoxin administration $[21,24,36]$. In the case of sepsis, the NO concentration is increased in 
exhaled air, thus acting as an early marker of lung inflammation $[16,31]$. We found that NO production is mainly from the lung. Furthermore, the extent of acute lung injury and the NO concentration in exhaled air are greatly reduced in lung perfused with physiological solution when compared to lung perfused with whole blood [24]. This finding suggests that cellular elements are essential to the production of $\mathrm{NO}$ and lung injury. These changes are attenuated by pretreatment with L-NAME, aminoguanidine and dexamethasone, suggesting that iNOS is involved in the pathogenesis of sepsis-induced PE. Exogenous and endogenous NO also causes lung injury following I/R [21].

In conscious and unrestrained rats following intravenous infusion of lipopolysaccharide (LPS; $10 \mathrm{mg} / \mathrm{kg}$ ), hypotension and plasma nitrite/nitrate reached a peak at 9-12 h. This finding indicates that increased NO release accounts for the systemic hypotension. Plasma amylase reached a peak at $18-24 \mathrm{~h}$ after LPS infusion. The increase in plasma amylase may be an indication of multiple organ damage in the late stage of sepsis. Postmortem examination of the lung revealed a slight increase in lung weight compared to normal lung. Lung injury was mild [25]. Higher doses of LPS may be required to induce severe PE, and changes in biochemical factors may be used to elucidate the pathogenesis of acute lung injury in this conscious rat model.

Our recent report of ARDS patients suggests that cerebral compression, air embolism, I/R and endotoxin are not the only causes of ARDS [19]. In patients with Japanese $B$ encephalitis, viral destruction of the depressor areas may induce central sympathetic activation resulting in ARDS. Disruption of a cerebral aneurysm can cause severe intracranial hypertension and ARDS. Obstruction of lymphatic drainage can also lead to severe ARDS. In fat embolism, biochemical mediators such as cGMP, NO and serotonin may be involved in the pathogenesis of ARDS.

\section{Conclusion and Suggestions}

A series of studies on centrogenic PE clearly suggest that massive sympathetic drive causes intensive sympathetic vasoconstriction. The blood is shifted from mainly the splanchnic beds to the lung. Additional pulmonary vasoconstriction adds to the disruption of pulmonary small vessels in the face of a volume-distended lung. In isolated perfused lung, PE induced by air embolism, I/R and endotoxin is mainly mediated by NO. Patients with
Japanese B encephalitis demonstrate viral destruction of the medullary depressor areas. The release of sympathetic drive may be the cause of ARDS due to viral lesions in the brain. Chang et al. [1] reported that patients with enterovirus infection demonstrated hand, foot and mouth lesions, and in the most dramatic case, ARDS ensued. Unfortunately, pathological examination of the brain was not reported in their study. The outbreaks of SARS from early to mid-2003 resulted in high mortality. The mechanism of coronavirus-induced SARS is still unknown. It may be interesting to see whether coronavirus and/or enterovirus introduced into the animal can produce brain lesions as observed in patients with Japanese B encephalitis. A recent observation by $\mathrm{Hsu}$ et al. [unpubl. data] revealed that ARDS occurred after rabies infection as a consequence of a brain lesion similar to that seen in Japanese $B$ encephalitis.

Research with isolated lung used NO substrate and blockers to elucidate the detrimental role of NO in the acute injury caused by air embolism, I/R and endotoxin. We are now extending this research with the measurement of biochemical factors and expression of mRNA for cytokines and NOS.

In the conscious rat model, investigation of dosedependent changes in physiological parameters, biochemical factors and pathological changes should be carried out in order to observe dose-dependent organ injury, including that in the lung.

Experimental results in animals with chronic deprivation of constitutive NOS and iNOS and with NO knock-in or knock-out may further elucidate the involvement of different NOS forms in the pathogenesis of ARDS.

\section{Acknowledgements}

The authors are grateful to the National Science Council of Taiwan, Shin Kong Wu Ho-Su Memorial Foundation for financial support. We acknowledge the assistance of C.C. Chang in preparation of the manuscript. 


\section{References}

1 Chang LY, Huang YC, Lin TY. Fulminant neurogenic pulmonary edema with hand, foot and mouth disease. Lancet $352: 367-368 ; 1998$.

2 Chen HI. Circulatory changes underlying the Cushing reaction. Natl Sci Counc Mon 16: 1489-1505; 1988 .

3 Chen HI. Hemodynamic mechanisms of neurogenic pulmonary edema. Biol Signals 4:186$192 ; 1995$

4 Chen HI, Chai CY. Pulmonary edema and hemorrhage as a consequence of systemic vasoconstriction. Am J Physiol 227:144-151;1974.

5 Chen HI, Hu CT. Endogenous nitric oxide on arterial hemodynamics: A comparison between normotensive and hypertensive rats. Am J Physiol 273:H1816-H1823;1997.

6 Chen HI, Hu CT, Wu CY, Wang D. Nitric oxide in systemic and pulmonary hypertension. J Biomed Sci 4:244-248;1997.

7 Chen HI, Huang HS, Yang JG, Wang D. Vasodilator and oxidant scavenger in the neurogenic pulmonary edema induced by cerebral compression. Chin J Physiol 35:123-131:1992.

8 Chen HI, Liao TF, Kuo L, Ho ST. Centrogenic pulmonary hemorrhagic edema induced by cerebral compression in rats: Mechanism of volume and pressure loading in the pulmonary circulation. Circ Res 47:366-373;1980.

9 Chen HI, Lin JD, Chai CY. Pulmonary hemorrhagic edema induced by cerebral compression: Blood volume shift from the systemic circulation to the lungs. Proc Natl Sci Counc B 4:381$386 ; 1980$.

10 Chen HI, Lin JD, Liao TF. Participation of regional sympathetic outflows in the centrogenic pulmonary pathology. Am J Physiol 240: H109-H115;1981.

11 Chen HI, Shih WJ, Chen TP. A scintiphotographic study of pulmonary edema and hemorrhage induced by cerebral compression and norepinephrine. Chin J Physiol 22:65-72; 1976.

12 Chen HI, Sun SC, Chai CY. Pulmonary edema and hemorrhage resulting from cerebral compression. Am J Physiol 224:223-229;1973.

13 Chen HI, Wang DJ. Systemic and pulmonary hemodynamic responses to intracranial hypertension. Am J Physiol 247:H715-H721;1984.

14 Chen HI, Wang YC, Chai CY. The Cushing responses in the systemic and pulmonary circulation: The role of adrenal glands, bronchial circulation and pulmonary innervation. Chin $\mathbf{J}$ Physiol 30:87-101;1987.
15 Fell C, Rushmer RF. Anatomic distribution of induced changes in blood volume, evaluated by regional weighing. $J$ Appl Physiol 16:85-88; 1961.

16 Gustafsson LE, Leone AM, Presson MG, Wiklund NP, Moncada S. Endogenous nitric oxide is present in the exhaled air of rabbits guinea pigs and humans. Biochem Biophys Res Commun 181:852-857;1991.

17 Hsu K, Wang D, Chang ML, Wu CP, Chen HI. Pulmonary edema induced by phorbol myristate acetate is attenuated by compounds that increase intracellular cAMP. Res Exp Med 196:17-28;1996.

18 Hsu K, Wang D, Wu SY, Shen CY, Chen HI. Ischemia-reperfusion lung injury attenuated by $\mathrm{ATP}-\mathrm{MgCl}_{2}$ in rats. $J$ Appl Physiol 76:545$552 ; 1994$.

19 Hsu YH, Kao SJ, Lee RP, Chen HI. Acute pulmonary oedema: Rare causes and possible mechanisms. Clin Sci 104:259-264;2003.

$20 \mathrm{Hu}$ CT, Chang KC, Wu CY, Chen HI. Acute effects of nitric oxide blockade with L-NAME on arterial hemodynamics in the rat. BrJ Pharmacol 122:1237-1243;1997.

21 Kao JS, Peng TC, Lee RP, Hsu K, Chen CF, Huang YK, Wang D, Chen HI. Nitric oxide mediates lung injury induced by ischemiareperfusion in rats. $\mathrm{J}$ Biomed Sci 10:58-64; 2003.

22 Ksiazek TG, Erman D, Goldsmith CS, Zaki SR, Peret T, Emery S, Tong S, Urbani C, Comer JA, Lim W, Rollin PE, Dowell SF, Ling AE, Humphrey CD, Shieh WJ, Guarner J, Paddock CD, Rota P, Fields B, DeRisi J, Yang JY, Cox N, Hughes JM, LeDuc JW, Bellini WJ, Anderson LJ, and the SARS working group. A novel coronavirus associated with severe acute respiratory syndrome. N Engl J Med 348:19531966:2003.

23 Lee N, Hui D, Wu A. A major outbreak of severe acute respiratory syndrome in Hong Kong. N Engl J Med 348:1984-1992;2003.

24 Lee RP, Wang D, Kao SJ, Chen HI. The lung is the major site that produces nitric oxide to induce acute pulmonary oedema in endotoxin shock. Clin Exp Pharmacol Physiol 28:315320;2001.

25 Lee RP, Wang D, Lin NT, Chen HI. Physiological and chemical indicators for early and late stages of sepsis in conscious rats. $\mathbf{J}$ Biomed Sci 9:613-621;2002.

26 Lee RP, Wang D, Lin NT, Chou YW, Chen HI: A modified technique for tail cuff pressure measurement in unrestrained conscious rats. $J$ Biomed Sci 9:424-427;2002.
27 Moncada S, Palmer RMJ, Higgs EA. Nitric oxide: Physiology, pathophysiology and pharmacology. Pharmacol Rev 43:109-142;1991.

28 Poutanen SM, Low DE, Henry B. Identification of severe acute respiratory syndrome in Canada. N Engl J Med 348:1993-2003;2003.

29 Shen CY, Wang D, Chang ML, Hsu K. Protective effect of mepacrine on hypoxia-reoxygenation-induced acute lung injury in rats. $J$ Appl Physiol 78:225-231;1995.

30 Stamler JS, Loh E, Roddy MA, Currie KE, Creager MA. Nitric oxide regulates basal systemic and pulmonary vascular resistance in healthy humans. Circulation 89:2035-2040; 1994.

31 Stewart TE, Valenza F, Ribeiro SP, Wener AD, Volgyesi G, Mullen JB, Slutsky AS. Increased nitric oxide in exhaled gas as an early marker of lung inflammation in a model of sepsis. Am J Respir Crit Care Med 151:713-718;1995.

$32 \mathrm{Su}$ CF, Hu CT, Chen HI. Effects of intracranial hypertension on steady and pulsatile hemodynamics in dogs. Clin Exp Pharmacol Physiol 26:898-902;1999

33 Szabo C, Mitchell JA, Thiemermann C, Vane JR. Nitric oxide mediated hyporeactivity to noradrenaline precedes the induction of nitric oxide synthase in endotoxin shock. $\mathrm{Br} J$ Pharmacol 108:786-792;1993.

34 Tsang KW, Ho PL, Ooi GC, Yee WK, Wang T, Chan-Yeung M, Lam WK, Seto WH, Yam LY, Cheung TM, Wong PC, Lam B, Ip MS, Chan J, Yuen KY, Lai KN. A cluster of cases of severe acute respiratory syndrome in Hong Kong. N Engl J Med 348:1975-1983;2003.

35 Wang D, Hsu K. Hwang CP, Chen HI. Measurement of nitric oxide release in the isolated perfused rat lung. Biochem Biophys Res Commun 208:1016-1020;1995.

36 Wang D, Li MH, Hsu K, Shen CY, Chen HI Air embolism-induced lung injury in isolated rat lungs. J Appl Physiol 72:1235-1242;1992.

37 Wang D, Wei J, Hsu K, Jau JC, Lieu MW, Chao TJ, Chen HI. Effects of nitric oxide synthase inhibitors on systemic hypotension, cytokines and inducible nitric oxide synthase expression and lung injury following endotoxin administration in rats. J Biomed Sci 6:28-35; 1999.

38 Wright CE, Rees DD, Moncada S. Protective and pathological roles of nitric oxide in endotoxin shock. Cardiovasc Res 26:48-57;1992. 